\title{
THE ORIGIN OF THE ECCENTRICITIES OF THE RINGS OF URANUS
}

\author{
PETER GOLDREICH \\ California Institute of Technology \\ AND \\ ScotT Tremaine \\ Institute for Advanced Study, Princeton \\ Received 1980 July 7; accepted 1980 August 13
}

\begin{abstract}
We consider the effect of gravitational perturbations from a nearby satellite on the eccentricity $e$ of a narrow particulate ring. The perturbations near a resonance in an eccentric ring may be divided into corotation and Lindblad terms. For small $e$, the corotation terms damp $e$, whereas the Lindblad terms excite $e$. In the absence of saturation the corotation terms win by a small margin, and $e$ damps. However, if the perturbations open gaps at the strongest resonances, then the Lindblad terms win, and $e$ grows. This result offers an explanation for the existence of both circular and eccentric rings around Uranus.

We also show that eccentricity changes induced by circular rings on eccentric satellite orbits are similar to those induced by satellites with circular orbits on eccentric rings.
\end{abstract}

Subject headings: planets: satellites - planets: Uranus

\section{INTRODUCTION}

One of the most remarkable features of the Uranian ring system is that several of the rings are essentially circular, while others are clearly eccentric. The most striking example of an eccentric ring is the $\epsilon$ ring, with $e=0.0078$ (Nicholson $e t$ al. 1978). Goldreich and Tremaine (1979a, here GT3) argued that the rings are constrained against spreading, because of interparticle collisions and radiation drag, by repulsive gravitational torques from nearby small satellites. In this paper we investigate the effect of the gravitational perturbations from such hypothetical satellites on the ring eccentricities. We provide a natural explanation in terms of these perturbations for the existence of both circular and eccentric rings.

In $\S$ II we define our notation and estimate the decay time scale of the eccentricity of an unconstrained ring. Section III discusses the types of resonance which are present in a ring perturbed by a satellite. Section IV contains the potential theory needed for our applications. In $\S \mathrm{V}$ we derive formulae which describe the evolution of the eccentricity of either $(a)$ a satellite near a circular ring or $(b)$ a ring near a satellite on a circular orbit. Application of our theory to the $\epsilon$ ring is made in § VI. Finally, § VII contains a summary and guide to the principal results. The reader who is mainly interested in applications may read only $\S$ II, VI, and VII.

We refer to some of our earlier papers on rings (Goldreich and Tremaine 1978a, 1978b, 1979a, 1979b, 1979c, 1980) as GT1, ..., GT6.

\section{PRELIMINARIES}

\section{a) Notation}

We consider orbits in the equatorial plane of a central body of mass $M$. Position in this plane is measured by rectangular $(x, y)$ or polar $(r, \theta)$ coordinates. A complete set of orbital elements is: $a=$ semimajor axis; $e=$ eccentricity; $\varpi=$ longitude of periapse (measured from $\theta=0) ; f=$ true anomaly. We also use the auxiliary elements: $n=$ mean motion, where $n^{2} a^{3}=G M ; I=(G M a)^{1 / 2}\left[1-\left(1-e^{2}\right)^{1 / 2}\right]=$ radial action; $\lambda=$ mean longitude; $h=e \sin \varpi$; $k=e \cos \varpi$. In later sections we consider perturbations due to a satellite of mass $m_{s}$ and orbital elements $a_{s}, e_{s}, \varpi_{s}$, etc. Except for a brief discussion in $\S$ II $c$, we neglect the oblateness of the planet and approximate it as a point mass.

\section{b) Conservation Laws for a Ring of Colliding Particles}

An isolated ring evolves slowly as a result of particle collisions. The conservation of linear momentum yields two constraints on the collisional evolution of the orbital elements of the ring.

1. Consider two particles with orbital elements $a_{1}, e_{1}$ and $a_{2}, e_{2}$. For simplicity we assume that their masses are equal. Conservation of the tangential component of linear momentum implies that the total orbital angular momentum of the particles is conserved (we neglect changes in the particles' spins).

$$
\Delta_{c}\left[a_{1}{ }^{1 / 2}\left(1-e_{1}{ }^{2}\right)^{1 / 2}+a_{2}{ }^{1 / 2}\left(1-e_{2}{ }^{2}\right)^{1 / 2}\right]=0,
$$

where $\Delta_{c}$ denotes the change due to a collision. 
2. Consider the change in the eccentricity vectors $e_{i}=k_{i} \hat{x}+h_{i} \hat{y}, i=1,2$ during the collision. From Gauss's form of the perturbation equations,

$$
\begin{aligned}
& \Delta_{c} h_{i}=\frac{\left(1-e_{i}{ }^{2}\right)^{1 / 2}}{n_{i} a_{i}}\left\{-R_{i} \cos \theta+S_{i}\left[1+\frac{r}{a_{i}\left(1-e_{i}^{2}\right)}\right] \sin \theta+\frac{r S_{i} h_{i}}{a_{i}\left(1-e_{i}{ }^{2}\right)}\right\}, \\
& \Delta_{c} k_{i}=\frac{\left(1-e_{i}{ }^{2}\right)^{1 / 2}}{n_{i} a_{i}}\left\{R_{i} \sin \theta+S_{i}\left[1+\frac{r}{a_{i}\left(1-e_{i}{ }^{2}\right)}\right] \cos \theta+\frac{r S_{i} k_{i}}{a_{i}\left(1-e_{i}{ }^{2}\right)}\right\} .
\end{aligned}
$$

Here $R_{i}$ and $S_{i}$ are the radial and tangential specific impulses felt by particle $i$. Equations (2) are only valid when $R_{i}$, $S_{i} \ll n_{i} a_{i}$. There are no subscripts on $r$ and $\theta$ in equation (2) because the collision is assumed to take place at a point (i.e., we neglect the particle size). Since the masses of the particles are assumed to be equal, we have $R_{1}+R_{2}=0$ and $S_{1}+S_{2}=0$. Thus

$$
\begin{aligned}
\Delta_{c} h_{1} & +\Delta_{c} h_{2}=\left(S_{1} \sin \theta-R_{1} \cos \theta\right)\left[\frac{\left(1-e_{1}{ }^{2}\right)^{1 / 2}}{n_{1} a_{1}}-\frac{\left(1-e_{2}{ }^{2}\right)^{1 / 2}}{n_{2} a_{2}}\right] \\
& +r S_{1} \sin \theta\left[\frac{1}{n_{1} a_{1}{ }^{2}\left(1-e_{1}{ }^{2}\right)^{1 / 2}}-\frac{1}{n_{2} a_{2}{ }^{2}\left(1-e_{2}{ }^{2}\right)^{1 / 2}}\right]+r S_{1}\left[\frac{h_{1}}{n_{1} a_{1}{ }^{2}\left(1-e_{1}{ }^{2}\right)^{1 / 2}}-\frac{h_{2}}{n_{2} a_{2}{ }^{2}\left(1-e_{2}{ }^{2}\right)^{1 / 2}}\right],
\end{aligned}
$$

with a similar expression for $\Delta_{c} k_{1}+\Delta_{c} k_{2}$. In a ring with random velocities of order $c$, typically $\left(a_{1}-a_{2}\right) / a_{1} \sim$ $\left(e_{2}-e_{1}\right) \sim\left(h_{2}-h_{1}\right) \sim\left(k_{2}-k_{1}\right) \sim O(c / n a)$. The specific impulses $R_{i}$ and $S_{i}$ are $O(c)$. Thus the changes in total eccentricity vector are

$$
\Delta_{c}\left(e_{1}+e_{2}\right)=\hat{x} \Delta_{c}\left(h_{1}+h_{2}\right)+\hat{y} \Delta_{c}\left(k_{1}+k_{2}\right) \sim O\left[(c / n a)^{2}\right] .
$$

Since $c / n a \ll 1$ for planetary rings, the changes are very small. For comparison the change in the eccentricity vector of an individual ring particle is $\Delta_{c} e_{i} \sim O(c / n a)$, which is much larger. These considerations lead us to define the mean eccentricity vector of a ring of $N$ particles as $\langle e\rangle=N^{-1} \sum_{i=1}^{N} e_{i}$. The change in $\langle e\rangle$ per collision is $O\left[N^{-1}(c / n a)^{2}\right]$. The average rate of change depends on whether independent collisions lead to correlated or uniorrelated changes in $\langle e\rangle$. The most rapid changes would occur in the former case; in this case $\langle e\rangle$ evolves on a time scale $t_{e} \sim t_{\text {coll }}(n a / c)^{2} e$, where $t_{\text {coll }}$ is the collision time. Even this time scale is, in general, sufficiently long $\left(\sim 10^{10}\left[0.01 \mathrm{~cm} \mathrm{~s}^{-1} c^{-1}\right]^{2}\right.$ yr for the $\epsilon$ ring, using the parameters of $\S \mathrm{VI})$ that $\langle\boldsymbol{e}\rangle$ can be considered to be conserved exactly under collisions for the purposes of this paper. We use this conservation law in $\S$ IIId.

\section{c) The Survival of an Elliptical Ring}

In the preceding subsection we showed that the circularization time for an eccentric ring due to collisions is $t_{e} \gtrsim t_{\text {coll }}(n a / c)^{2} e$. If the ring surrounds an oblate planet, differential precession may lead to circularization in a much shorter time.

Consider a set of particles with identical eccentricity vectors $e_{0}=k_{0} \hat{x}+h_{0} \hat{y}$, uniformly spread over a range of semimajor axes $a_{0}-\Delta a / 2$ to $a_{0}+\Delta a / 2$. Assume that the optical depth in the ring is of order unity and that random motions are negligible. Then, if the particles are started from periapse at the same time, their trajectories will intersect near apoapse as a result of differential precession. In a ring with optical depth $\tau \gtrsim 1$ collisions occur when the trajectories intersect. At this time the mean eccentricity vector takes the form

and its magnitude is

$$
\langle e\rangle=e_{0}\langle\cos \Delta \varpi\rangle+\left(\hat{y} k_{0}-\hat{x} h_{0}\right)\langle\sin \Delta \varpi\rangle
$$

$$
e^{\prime}=|\langle e\rangle|=e_{0}\left(\langle\cos \Delta \varpi\rangle^{2}+\langle\sin \Delta \varpi\rangle^{2}\right)^{1 / 2} .
$$

Here $\Delta \dot{\varpi}=\pi \varpi / n\left(a_{0}\right)$ and $\dot{\varpi}=\frac{3}{2} J_{2} R^{2}(G M)^{1 / 2} a^{-7 / 2}$. The latter is the precession rate due to the planet's dynamical oblateness $J_{2}$. If $\Delta a \ll a$, we have

$$
e^{\prime} \approx e_{0}\left[1-\frac{1}{24}\left(\frac{d \Delta \varpi}{d a} \Delta a\right)_{a_{0}}^{2}\right]=e_{0}\left[1-\frac{1}{24}\left(\frac{\pi}{n} \frac{d \dot{\varpi}}{d a} \Delta a\right)_{a_{0}}^{2}\right] .
$$

Thus, the magnitude of the mean eccentricity decays on a time scale

$$
t_{d}=\frac{24 n}{\pi}\left(\frac{d \dot{\varpi}}{d a} \Delta a\right)^{-2} \text {. }
$$

For the $\epsilon \operatorname{ring} t_{d} \sim 4 \times 10^{7} \mathrm{yr}$, which is short compared to the age of the solar system. Note that in a ring with $\tau \ll 1, t_{d}$ is shorter because the change in apse angle $\Delta \varpi$ is larger between collisions.

We have suggested (GT5) that precession due to the self-gravity of the $\epsilon$ ring can cancel the differential precession due to the planet, so that $t_{d} \rightarrow \infty$. However, this model does not explain why the Uranian system contains both circular and 
eccentric rings. To investigate this we must consider the influence of gravitational perturbations from nearby satellites on the growth and decay of the ring eccentricity.

\section{RESONANCES IN RINGS}

a) Noninteracting Particles

The perturbing potential due to a satellite with mean motion $n_{s}$ may be written as the sum of terms of the form

$$
\begin{aligned}
\phi & =\phi_{m k}(a, e) \cos \Phi_{m k}, \\
\Phi_{m k} & =k \lambda+(m-k) \varpi-\omega t,
\end{aligned}
$$

where $k, m$ are integers, ${ }^{1} m \geq 0, \lambda$ is the mean longitude, $\phi_{m k}$ is real, and $\phi$ is evaluated at the position of a particle with orbital elements $(a, e, \lambda, \varpi)$. The validity of equation (9) requires particular choices for the origin of $t$ and $\theta ;$ namely, $\theta=0$ is the satellite periapse, and $t=0$ is the epoch of periapse passage. This is not an important restriction. In general,

$$
\omega=\ln _{s},
$$

where $l$ is an integer. If $e_{s}=0$, then $l=m$. The form of $\phi_{m k}$ is not needed in this section; it is determined in $\S$ IV.

The orbital perturbations forced by the $(m, k)$ term are largest near the resonant semimajor axis $a_{0}$ defined by

$$
n_{0}=n\left(a_{0}\right)=\omega / k \text {. }
$$

The equations of motion under the perturbation (eq. [9]) are simplified if we replace the eccentricity $e$ by the radial action

$$
I=n a^{2}\left[1-\left(1-e^{2}\right)^{1 / 2}\right] .
$$

Note that for $e \ll 1, I \approx \frac{1}{2} n a^{2} e^{2}$. The equations of motion are

$$
\begin{aligned}
& \frac{d a}{d t}=-\frac{2}{n a} \frac{\partial \phi}{\partial \lambda}, \\
& \frac{d I}{d t}=\frac{\partial \phi}{\partial \varpi}, \\
& \frac{d \varpi}{d t}=-\frac{\partial \phi}{\partial I}, \\
& \frac{d \lambda}{d t}=n+\frac{2}{n a} \frac{\partial \phi}{\partial a} .
\end{aligned}
$$

It $\phi$ has the form in equation (9), there are two integrals of the motion:

$$
\begin{aligned}
& C_{1}=-\frac{1}{2} n^{2} a^{2}+\phi-n a^{2} \omega / k, \\
& C_{2}=n a^{2}(k-m)-k I .
\end{aligned}
$$

We examine the behavior of the solutions for $k=m$ and $k \neq m$ separately.

First consider resonances with $k=m$. For any resonance the perturbation potential (eq. [9]) is stationary in a frame rotating with the " pattern speed" $\omega / m$. When $k=m$, the resonant angular speed $n_{0}=\omega / m$ (eq. [11]). Thus, the resonance occurs where a particle corotates with the pattern. Hence, resonances with $k=m$ are called "corotation" resonances. For these resonances $C_{2}=-m I$. Since the perturbation is small, we evaluate $\phi_{m k}$ in equation (14a) at $a_{0}$ (eq. [11]) and expand the unperturbed terms in $C_{1}$ to the lowest nonvanishing order in $\Delta a=a-a_{0}$. Neglecting the unimportant constant term,

$$
C_{1} \approx-\frac{3}{8} n_{0}^{2} \Delta a^{2}+\phi_{m m}\left(a_{0}, I\right) \cos \Phi_{m m}
$$

A plot of the contours of $C_{1}$ in the $\left(\Phi_{m m}, \Delta a\right)$-plane reveals that stationary solutions exist with $\Phi_{m m}=0, \pi$. If $\phi_{m m}>0$, the solution with $\Phi_{m m}=0$ is dynamically stable (it is located at an extremum of $C_{1}$ ), while the solution with $\Phi_{m m}=\pi$ is unstable (it is located at a saddle point). If $\phi_{m m}<0$, the stability characteristics of the stationary solutions are reversed. Solutions near the stable stationary solution represent libration of $\Phi_{m m}$. For small amplitude, the libration frequency is

\footnotetext{
${ }^{1}$ The integer $k$ should not be confused with the component $k$ of the eccentricity vector $(h, k)$. The latter meaning is not used in the remainder of this paper.
} 
$\left(3 m^{2}\left|\phi_{m m}\right| / a_{0}^{2}\right)^{1 / 2}$. Libration occurs if $C_{1}>-\left|\phi_{m m}\right|$. If $C_{1}<-\left|\phi_{m m}\right|$, circulation takes place. The maximum change in semimajor axis for librating orbits is

$$
\Delta a_{r}=8\left(\frac{\phi_{m m}}{3 n_{0}{ }^{2}}\right)^{1 / 2} .
$$

The variable $\Delta a_{r}$ can be regarded as a measure of the "width" of the resonance.

If $k \neq m$, the nature of the solution depends upon the size of the eccentricity $e$. For $e \ll 1, \phi_{m k} \propto I^{|k-m| / 2} \propto e^{|k-m|}$. Thus, if $e$ is sufficiently small, changes in $e$ caused by the perturbation may have important effects of the motion.

Let us write $\phi_{m k}\left(a_{0}, I\right)=I^{|k-m| / 2} \psi_{m k}$ and set $\Delta a=a-a_{0}$ as before. The integrals (eq. [14]) are approximately

$$
\begin{aligned}
& C_{1}=-\frac{3}{8} n_{0}{ }^{2} \Delta a^{2}+I^{|k-m| / 2} \psi_{m k} \cos \Phi_{m k}, \\
& C_{2}=\frac{1}{2}(k-m) n_{0} a_{0} \Delta a-k I .
\end{aligned}
$$

Denote the initial values of $\Delta a$ and $I$ by $\Delta a_{i}$ and $I_{i}$ and write $\delta a=\Delta a-\Delta a_{i}, \delta I=I-I_{i}$. In some cases $|\delta a|$ and $|\delta I|$ can be as large as $\left|\Delta a_{i}\right|$ and $I_{i}$ during the particle's trajectory. However, for our purposes it is sufficient to consider two limiting cases: (1) $|\delta a| \ll\left|\Delta a_{i}\right| ;(2)|\delta I| \ll I_{i}$. The important resonances in planetary rings usually fall into one of these two categories.

Case (1): $|\delta a| \ll\left|\Delta a_{i}\right|$. We write equations (17) as

$$
\begin{aligned}
& C_{1}=-\frac{3}{4} n_{0}^{2} \Delta a_{i} \delta a+I^{|k-m| / 2} \psi_{m k} \cos \Phi_{m k}, \\
& C_{2}=\frac{1}{2}(k-m) n_{0} a_{0} \delta a-k I .
\end{aligned}
$$

These equations yield a third constant of the motion,

$$
C_{3}=\frac{3 n_{0} I}{2 a_{0}}-\left(\frac{k-m}{k}\right) I^{|k-m| / 2} \frac{\psi_{m k}}{\Delta a_{i}} \cos \Phi_{m k} .
$$

We are interested in planetary rings, for which the perturbing potential $\psi_{m k}$ due to a satellite is very small. For $|k-m| \geq 2$, the second term in equation $(19)$ is generally small in comparison to the first term; thus $I \approx$ constant. However, for $|k-m|=1$, the second term always dominates the first term as $I \rightarrow 0$. Consequently, resonances with $|k-m|=1$ force a substantial eccentricity on an initially circular orbit, whereas resonances with $|k-m|>1$ cannot. Resonances with $|k-m|=1$ are called Lindblad resonances; they are much more important than resonances with $|k-m|>1$ in circular rings because they can excite eccentricity.

According to equation (19) there are stationary solutions near a Lindblad resonance when

$$
\cos \Phi_{m k}= \pm 1, \quad I_{r}{ }^{1 / 2}= \pm\left(\frac{k-m}{3 k}\right) \frac{a_{0} \psi_{m k}}{n_{0} \Delta a_{i}}, \quad|k-m|=1,
$$

where the \pm sign is chosen so that $I_{r}{ }^{1 / 2}>0$. Orbits librate if $C_{3}<0$ and circulate if $C_{3}>0$. For small amplitudes, the libration frequency is $\left|3 k n_{0} \Delta a_{i} / 2 a_{0}\right|$. The maximum libration amplitude is $\Delta \Phi_{m k}=\pi$, in contrast to the corotation resonance where $\Phi_{m m}$ can librate through $2 \pi$. The maximum eccentricity in a librating orbit is given by the relation $I_{\max }=4 I_{r}$. The eccentricity in a circulating orbit varies between limits given by $I_{1}$ and $I_{2}$, where

$$
I_{1}{ }^{1 / 2}-I_{2}^{1 / 2}=2 I_{r}{ }^{1 / 2} \text {. }
$$

Thus, orbits with $I_{i} \lesssim I_{r}$ (eq. [20]) will generally suffer large eccentricity changes, whereas those with $I_{i} \gg I_{r}$ will retain roughly constant eccentricity (these orbits may also be treated by the analysis described below for the case $\left|\delta_{1}\right| \ll I_{i}$ ).

As stated above, these results are based on the approximation that $|\delta a| \ll\left|\Delta a_{i}\right|$; this is valid if

$$
\begin{aligned}
& \Delta a_{i}{ }^{3} \gg\left|\frac{k-m}{k}\right| \frac{a_{0} \psi_{k m}^{2}}{n_{0}{ }^{3}}, \quad I_{i} \lesssim I_{r}, \\
& \Delta a_{i}{ }^{2} \gg \frac{I_{i}{ }^{1 / 2}}{n_{0}{ }^{2}}\left|\psi_{m k}\right|, \quad I_{i} \gtrsim I_{r} .
\end{aligned}
$$

Case (2): $|\delta I| \ll I_{i}$. Here we can assume $I$ to be fixed at $I_{i}$ in equation (17)for $C_{1}$. From $C_{1}$ we see that the width of the resonance (defined as the maximum change in semimajor axis for a librating orbit) is

$$
\Delta a_{r}=8\left(\frac{1}{3 n_{0}{ }^{2}} I_{i}^{|k-m| / 2} \psi_{m k}\right)^{1 / 2} .
$$


The variable $\Delta a_{r}$ is the largest change in semimajor axis for any orbit. Thus, from $C_{2}$ we have $|\delta I|<$ $\frac{1}{2}|(k-m) / k| n_{0} a_{0} \Delta a_{r}$. For self-consistency we require $|\delta I| \ll I_{i}$ or

$$
I_{i}^{1-|k-m| / 4} \gg \frac{|k-m|}{|k|} a_{0} \psi_{m k}^{1 / 2}
$$

Whenever this condition is satisfied, changes in $I$ may be treated as small perturbations. The trajectory in the $\left(\Phi_{m k}, \Delta a\right)$-plane is approximately the same as that of the corotation resonance (see discussion after eq. [15]), but with additional small changes in $I$ given by $\delta I=\frac{1}{2}|(k-m) / k| n_{0} a_{0} \delta a$. Since these resonances only occur when the eccentricity exceeds a minimum value, we call them "eccentric" resonances.

All of these results on the orbits of particles near resonances are familiar from celestial mechanics. In the next section we examine the effects of interparticle collisions on these orbits.

\section{b) Colliding Particles \\ i) Corotation Resonances}

The simplest resonance to examine is the corotation $(k=m)$ resonance in a circular ring because the radial action $I$ is conserved at its unperturbed value $I=0$. Thus, the orbital radius $r=a$, and the resonant angle $\Phi_{m m}=m \lambda-$ $\omega t=m(\theta-\omega t / m)=m \theta_{\text {rot }}$ is simply the azimuthal angle $\theta_{\text {rot }}$ in a coordinate frame rotating with the pattern speed $\omega / m$. There is a one-to-one mapping between the dynamical variables $\Phi_{m m}, a$ and the spatial variables $\theta_{\text {rot }}, r$. For $I=0$, the level lines of the integral $C_{1}$ on the $\left(\Phi_{m m}, a\right)$ phase plane are congruent to the particle trajectories in real space. Since the level lines do not cross, these particle trajectories do not intersect even in a ring with high particle density.

In a ring composed of particles of finite size and mass, physical collisions and gravitational deflections always produce finite random motions (i.e., nonzero values of $I$ ). Collisions endow the ring with an effective kinematic viscosity $v$. Each particle random walks a radial distance $\sim \Delta a$ in a time $\Delta a^{2} / v$. Librations only occur if the diffusion time across $\Delta a_{r}$ (cf. eq. [16]) exceeds the libration time (see discussion after eq. [15]). Therefore, librating particles are present if

$$
\phi_{m m} \gg\left(\frac{n_{0}^{2} a_{0} v}{m}\right)^{2 / 3} \text {. }
$$

Strong corotation resonances contain both circulating and librating particles. In weak resonances the potential is not strong enough to build up large perturbations before they are damped by collisions. In the latter case (i.e., where inequality [25] is not satisfied), the treatment based on integrals of the motion which we have used so far is not appropriate. A proper treatment would require the solution of the Boltzmann equation with an approximate collision term. However, a simple procedure which captures the essential effects of collisions is to add a damping term, described by a time constant $\gamma$, to the perturbation equations (13). Thus, if $X_{1}$ is the first-order perturbation in one of $a, I, \varpi, \lambda$, we add $-\gamma X_{1}$ to the right-hand side of equations (13a)-(13d), respectively. We have published more detailed models describing the effects of collisions on simple resonances (GT2, GT4); we compare our prior results to those obtained from this simple model whenever possible.

A rough estimate for the damping constant $\gamma$ is arrived at by the following argument. The scale size of the perturbation potential is roughly $a_{0} / \mathrm{m}$. The disturbance it forces in the ring is sheared by differential rotation; the shear reduces the scale by a factor $\Omega t$ in time $t$, to roughly $a_{0} /(m \Omega t)$. A disturbance of this scale size is damped in a time $t \approx\left[a_{0} /(m \Omega t)\right]^{2} / v$. Solving for $t$ and equating $t$ to $\gamma^{-1}$, we find

$$
\gamma \sim\left(\frac{m^{2} \Omega^{2} v}{a_{0}^{2}}\right)^{1 / 3}
$$

This value for $\gamma$ is equal to the damping constant obtained for more detailed models of the ring dynamics in equation (98) of GT6.

Using the damping coefficient $\gamma$ we compute the secular torque exerted on the ring in the vicinity of a corotation resonance. We assume that libration does not occur (i.e., inequality [25] is violated) so that perturbation theory applies. The first-order perturbations in $a$ and $\lambda$ are given by

$$
\begin{aligned}
& \frac{d a_{1}}{d t}=-\frac{2}{n a} \frac{\partial \phi}{\partial \lambda}-\gamma a_{1}, \\
& \frac{d \lambda_{1}}{d t}=n_{1}+\frac{2}{n a} \frac{\partial \phi}{\partial a}-\gamma \lambda_{1} .
\end{aligned}
$$


The solutions are

$$
\begin{aligned}
& a_{1}=-\frac{2 m}{n a} \phi_{m m} \operatorname{Re}\left\{i \exp \left[i(m n-\omega) t-i m n t_{0}\right] /[i(m n-\omega)+\gamma]\right\}, \\
& \lambda_{1}=\frac{2}{n a} \frac{\partial}{\partial a} \phi_{m m} \operatorname{Re}\left\{\exp \left[i(m n-\omega) t-i m n t_{0}\right] /[i(m n-\omega)+\gamma]\right\},
\end{aligned}
$$

where $n\left(t-t_{0}\right)$ is the unperturbed mean longitude. The validity of the perturbation expansion requires $m \lambda_{1} \ll 1$ everywhere. If the damping time $\gamma$ is short (specifically, if $\gamma \gg m n\left|\partial \ln a / \partial \ln \phi_{m m}\right|$ ), then the maximum value of $m \lambda_{1} \approx m^{2}\left|\phi_{m m}\right| /(a \gamma)^{2}$. Using equation (26) we may show that the condition $m \lambda_{1} \ll 1$ reduces to the condition for the absence of librating particles. This result is not surprising since a librating particle generally has $m \lambda_{1} \approx 1$.

For $I=0$ the angular momentum of a particle is $J=(G M a)^{1 / 2}$. From equation (28a) there is no secular change in $J$ to first order. To second order

$$
\frac{d J_{2}}{d t}=-\frac{\partial^{2} \phi}{\partial \lambda^{2}} \lambda_{1}-\frac{\partial^{2} \phi}{\partial a \partial \lambda} a_{1} .
$$

We have not included a damping term in this equation because its only important effect is to remove the singularity in the variables $a_{1}$ and $\lambda_{1}$ at resonance. If we average $d J_{2} / d t$ over $t_{0}$, we obtain

$$
\left\langle\frac{d J_{2}}{d t}\right\rangle=\frac{\gamma m^{2}}{n a} \frac{\partial}{\partial a}\left[\frac{\phi_{m m}^{2}}{(m n-\omega)^{2}+\gamma^{2}}\right] .
$$

If $\gamma \ll m n$, we may write $\gamma /\left[(m n-\omega)^{2}+\gamma^{2}\right] \rightarrow \pi \delta(m n-\omega)$. Thus,

$$
\left\langle\frac{d J_{2}}{d t}\right\rangle=\frac{\pi m^{2}}{n a} \frac{\partial}{\partial a}\left[\phi_{m m}^{2} \delta(m n-\omega)\right] \text {. }
$$

If $\Sigma(a)$ is the ring surface mass density at radius $a$, the total torque on the ring is

$$
\frac{d J}{d t}=2 \pi \int \Sigma(a) a\left\langle\frac{d J_{2}}{d t}\right\rangle d a=-\frac{4}{3} m \pi^{2}\left[\frac{\phi_{m m}^{2} a}{n} \frac{d}{d a}\left(\frac{\Sigma}{n}\right)\right]_{m n=\omega} .
$$

This expression is valid if $\gamma \ll m n$ (so that the torque is concentrated near the resonance) and $\gamma \gg m^{2} \phi_{m m} / a_{0}^{2}$ (so that there are no librating particles and perturbation theory applies). In practice, the second constraint is more important; if it is violated (i.e., if inequality [25] is satisfied), then the torque is reduced below the value given by equation (32) because there is no secular torque on librating particles.

Equation (32) also represents the torque on a gaseous disk at the corotation resonance (Donner 1979; GT4). This result increases our confidence that the torque is independent of the details of the dissipation process.

The derivation given here is similar to the one used by Lynden-Bell and Kalnajs (1972) to determine the torques in disk galaxies. Their equation (30) can be reduced to our equation (32) (cf. GT4).

\section{ii) Lindblad Resonances}

The zero amplitude libration orbits near a Lindblad resonance have values of $\Phi_{m k}$ which differ by $\pi$ on opposite sides of the resonance. Thus, particles on one side of the resonance are at periapse, while particles at the same longitude on the other side are at apoapse. Unless there is a gap at the resonance, collisions must take place. We defer discussion of the case where a gap forms until later in this section. In the absence of a gap, departures from circular orbits may be treated by perturbation theory if the collision time is shorter than the libration time. This is the case for the known Uranian rings which have optical depths of order unity. Disks of low optical depth are discussed in GT6.

The angular momentum $J=(G M a)^{1 / 2}-I$. Just as for the corotation resonance, there is no secular change in $J$ to first order. To second order,

$$
\frac{d J_{2}}{d t}=-\left(\frac{\partial^{2} \phi}{\partial a \partial \varpi}+\frac{\partial^{2} \phi}{\partial a \partial \lambda}\right) a_{1}-\left(\frac{\partial^{2} \phi}{\partial \lambda \partial \varpi}+\frac{\partial^{2} \phi}{\partial \lambda^{2}}\right) \lambda-\left(\frac{\partial^{2} \phi}{\partial I \partial \varpi}+\frac{\partial^{2} \phi}{\partial I \partial \lambda}\right) I_{1}-\left(\frac{\partial^{2} \phi}{\partial \varpi^{2}}+\frac{\partial^{2} \phi}{\partial \varpi \partial \lambda}\right) \varpi_{1} .
$$

The quantities $a_{1}, \lambda_{1}, I_{1}, \varpi_{1}$ are determined by solving equations like equation (27) for the $(m, k)$ potential component. Averaging $d J_{2} / d t$ over the epoch of zero mean longitude $t_{0}$, we find

$$
\left\langle\frac{d J_{2}}{d t}\right\rangle=\gamma m\left[\frac{(k-m)}{2} \frac{\partial \phi_{m k}^{2} / \partial I}{(\omega-k n)^{2}+\gamma^{2}}+\frac{k}{n a} \frac{\partial}{\partial a} \frac{\phi_{m k}^{2}}{(\omega-k n)^{2}+\gamma^{2}}\right] .
$$


As before, we let $\gamma \rightarrow 0$ and consider circular rings, that is, $I \rightarrow 0$. Although equation (34) is valid for all $m, k$, we are interested in Lindblad resonances, which have $|m-k|=1$. For them $\phi_{m k} \propto e$ as $e \rightarrow 0$, so that $\phi_{m k}{ }^{2} \rightarrow 0$ and $\partial \phi_{m k}^{2} / \partial I \rightarrow$ constant. Thus

$$
\left\langle\frac{d J_{2}}{d t}\right\rangle=\frac{\pi m(k-m)}{2}\left(\frac{\partial \phi_{m k}^{2}}{\partial I}\right)_{I=0} \delta(\omega-k n), \quad|m-k|=1 .
$$

The total torque on the ring is

$$
\frac{d J}{d t}=2 \pi \int \Sigma(a) a\left\langle\frac{d J_{2}}{d t}\right\rangle d a=\frac{2 \pi^{2} m}{3 k}(k-m)\left(\frac{a^{2} \Sigma}{n} \frac{\partial \phi_{m k}^{2}}{\partial I}\right)_{I=0, \omega=k n}, \quad|m-k|=1 .
$$

Equation (36) is a special case of the Lindblad torque which Lynden-Bell and Kalnajs (1972) derived for disk galaxies (cf. GT4). It is also the correct formula for the secular torque on a gas disk due to the resonance (Donner 1979; GT4).

If the torque is sufficiently strong, it opens a gap in the ring near the resonance. The Lindblad torque could clear out a gap of radial width $\Delta r$ in a time

$$
t_{\mathrm{open}} \approx n^{2} \Delta r^{2} /\left(\partial \phi_{m k}^{2} / \partial I\right),
$$

whereas viscous diffusion would fill in the gap in $t_{\text {close }} \approx \Delta r^{2} / v$. A gap forms if $t_{\text {open }}<t_{\text {close }}$, or

$$
\frac{\partial \phi_{m k}^{2}}{\partial I}>n^{2} v \text {. }
$$

In the presence of a gap the torque on the ring is reduced below the value given by equation (36).

iii) Eccentric Resonances

In $\S$ III $a$ we found that motion near an eccentric resonance is similar to motion near a corotation resonance and consists of circulating and librating orbits in the $\left(\Phi_{m k}, \Delta a\right)$-plane. The difference is that the excursions in $a$ generate excursions in $I$ (through $C_{2}$, eq. [17]) and in $\varpi$ (through eq. [13c]). If we neglect variations in $I$ and $\varpi$, then at any time there is a one-to-one mapping between the $\left(\Phi_{m k}, \Delta a\right)$ phase plane and physical space. Thus, the nonintersection of trajectories in the phase plane implies that particle orbits do not cross, just as for the corotation resonance. Arguments similar to those leading to inequality (25) show that librating particles exist at an eccentric resonance if

$$
\phi_{m k} \gg\left(\frac{n_{0}^{2} a_{0} v}{m}\right)^{2 / 3} .
$$

By contrast, variations in radial action $I$ and periapse position $\varpi$ cause collisions and are efficiently damped. It is important to note that this damping does not affect the libration. To see this, suppose that $I$ and $\varpi$ are fixed at their initial values $I_{i}$ and $\varpi_{i}$. The equations of motion (13) become

$$
\begin{aligned}
& \frac{d a}{d t}=+\frac{2 k}{n a} \phi_{m k}\left(a, I_{i}\right) \sin \left[k \lambda+(m-k) \varpi_{i}-\omega t\right], \\
& \frac{d \lambda}{d t}=n+\frac{2}{n a} \frac{\partial \phi_{m k}}{\partial a}\left(a, I_{i}\right) \cos \left[k \lambda+(m-k) \varpi_{i}-\omega t\right] .
\end{aligned}
$$

These have the integral

$$
C_{1}=-\frac{1}{2} n^{2} a^{2}+\phi_{m k}\left(a, I_{i}\right) \cos \left[k \lambda+(m-k) \varpi_{i}-\omega t\right]-\frac{n a^{2} \omega}{k},
$$

which describes the trajectories in the $\left(\Delta a, \Phi_{m k}\right)$-plane. The damping of variations in $I$ and $\varpi$ does not damp libration or circulation in $\Delta a$ and $\Phi_{m k}$. If inequality (39) is satisfied, the formula for the second-order torque is

$$
\left(\frac{d J_{2}}{d t}\right)_{\mathrm{L}}=-\left(\frac{\partial^{2} \phi}{\partial I \partial \varpi}+\frac{\partial^{2} \phi}{\partial I \partial \lambda}\right) I_{1}-\left(\frac{\partial^{2} \phi}{\partial \varpi^{2}}+\frac{\partial^{2} \phi}{\partial \varpi \partial \lambda}\right) \varpi_{1},
$$

which is similar to our equation (33) except that the terms proportional to $a_{1}$ and $\lambda_{1}$ do not contribute since the periodic motions in $a$ and $\lambda$ are undamped. We call the terms arising from damped first-order variations in $I$ and $\varpi$ the " $L$ indblad " terms from the eccentric resonance, since these are the terms which yield the torque at a Lindblad resonance in a circular ring. This is the meaning of the subscript $L$ in equation (42).

Strictly, the terms in equation (42) should be evaluated along the librating or circulating orbit given by equation (41). However, the damping constant $\gamma$ is usually sufficiently large that the dominant contribution to the torque comes from 
circulating orbits far from the libration region. Hence, we evaluate each term along the unperturbed orbit. After averaging over epoch $t_{0}$, we find

$$
\left\langle\left(\frac{d J_{2}}{d t}\right)_{L}\right\rangle=\frac{\gamma}{2} m(k-m) \frac{\partial \phi_{m k}^{2} / \partial I}{(\omega-k n)^{2}+\gamma^{2}} .
$$

Letting $\gamma \rightarrow 0$ and integrating over the ring, we obtain the total torque due to Lindblad terms,

$$
\left(\frac{d J}{d t}\right)_{\mathrm{L}}=\frac{2 \pi^{2} m}{3 k}(k-m)\left(\frac{a^{2} \Sigma}{n} \frac{\partial \phi_{m k}^{2}}{\partial I}\right)_{I, \omega=k n}
$$

Note that equation (44) reduces to equation (36) as $I \rightarrow 0$.

This is the complete expression for the torque on the ring if inequality (39) is satisfied by a large margin. If the inequality is not satisfied, collisions are sufficiently strong to damp perturbations in $a$ and $\lambda$. Then there is an additional torque

$$
\left(\frac{d J_{2}}{d t}\right)_{C}=-\left(\frac{\partial^{2} \phi}{\partial a \partial \varpi}+\frac{\partial^{2} \phi}{\partial a \partial \lambda}\right) a_{1}-\left(\frac{\partial^{2} \phi}{\partial \lambda \partial \varpi}+\frac{\partial^{2} \phi}{\partial \lambda^{2}}\right) \lambda_{1} .
$$

We call these terms the "corotation" terms from the eccentric resonance since perturbations in $a$ and $\lambda$ characterize a corotation resonance. Averaging over $t_{0}$, we find

$$
\left\langle\left(\frac{d J_{2}}{d t}\right)\right\rangle_{c}=\frac{\gamma m k}{n a} \frac{\partial}{\partial a}\left[\frac{\phi_{m k}^{2}}{(\omega-k n)^{2}+\gamma^{2}}\right] .
$$

As usual, we let $\gamma \rightarrow 0$ and integrate over the ring. We obtain

$$
\left(\frac{d J}{d t}\right)_{C}=-\frac{4}{3} m \pi^{2}\left[\frac{\phi_{m k}^{2}}{n} a \frac{d}{d a}\left(\frac{\Sigma}{n}\right)\right]_{k n=\omega} .
$$

Note that equation (47) is identical to equation (32) for the torque at a corotation resonance if we set $m=k$.

To summarize, we have divided the torque at an eccentric resonance into a "corotation" torque and a "Lindblad" torque. If the perturbation potential is sufficiently weak, both torques have their unsaturated values. If librating orbits exist near the resonance, then the corotation torque is saturated. If a gap opens at the resonance, then both torques are reduced below their unsaturated values.

\section{c) The Effect of a Circular Ring on the Orbital Eccentricity of a Satellite}

To determine the effect of the gravitational back reaction of the ring on the satellite's orbital eccentricity $e_{s}$ we use the fact that gravitational interactions conserve total energy and total angular momentum. If $E_{s}$ and $J_{s}$ are the satellite's energy and angular momentum per unit mass, we have

$$
e_{s}^{2}=1+\frac{2 E_{s} J_{s}^{2}}{G^{2} M^{2}}
$$

Thus

$$
\frac{d e_{s}^{2}}{d t}=\frac{2 a_{s}\left(1-e_{s}^{2}\right)}{G M n}\left(\dot{E}_{s}-n_{s}\left(1-e_{s}^{2}\right)^{-1 / 2} \dot{J}_{s}\right) .
$$

It follows from equations (9) and (15) that

$$
\dot{E}=n(k / m) \dot{J}=\omega \dot{J} / m,
$$

where the second equality holds because, at the resonance, $k n=\omega$. If $m_{s}$ is the satellite mass and $m_{r}$ is the ring mass, we have $\dot{E}_{s}=-\left(m_{r} / m_{s}\right) \dot{E}, \dot{J}_{s}=-\left(m_{r} / m_{s}\right) \dot{J}$. Thus,

$$
\frac{d e_{s}^{2}}{d t}=\frac{2\left(1-e_{s}^{2}\right) a_{s}}{G M}\left[n_{s}\left(1-e_{s}^{2}\right)^{-1 / 2}-\frac{\omega}{m}\right] \dot{J} .
$$

The variable $\dot{J}$ is obtained from equations (31) and (35) for corotation and Lindblad resonances, respectively. The sum over all resonances yields

$$
\begin{aligned}
\frac{d e_{s}^{2}}{d t}= & \frac{2 \pi a_{s}\left(1-e_{s}^{2}\right)}{G M}\left(\frac{m_{r}}{m_{s}}\right)\left(\frac{n_{s}}{n a^{2}}\right) \sum_{k} \llbracket\left[\left[k\left(1-e_{s}^{2}\right)^{-1 / 2}-\frac{\omega}{n_{s}}\right] k a \frac{\partial}{\partial a}\left[\phi_{k k}^{2} \delta(k n-\omega)\right]\right. \\
& +\frac{n a^{2}}{2} \delta(k n-\omega)\left\{\left[(k-1)\left(1-e_{s}^{2}\right)^{-1 / 2}-\frac{\omega}{n s}\right] \frac{\partial \phi_{k-1, k}^{2}}{\partial I}-\left[(k+1)\left(1-e_{s}^{2}\right)^{-1 / 2}-\frac{\omega}{n_{s}}\right] \frac{\partial \phi_{k+1, k}^{2}}{\partial I}\right\} \|_{I=0}
\end{aligned}
$$


We must also sum equation (52) over all values of $\omega$. Since the satellite motion is periodic with period $2 \pi / n_{s}$, the values of $\omega$ are restricted to $\omega=l_{s}$, where $l$ is an integer. We denote the component of $\phi_{m k}$ with $\omega=l_{s}$ by $\phi_{l m k}$. This notation is consistent with that of GT6, where only components with $m=k$ were used, which were denoted by $\phi_{l, m}^{s}$. For $m=0, \phi_{l 0 k}$ and $\phi_{-l, 0,-k}$ represent the same perturbation. We resolve this ambiguity by adopting the convention that $\phi_{l 0 k}=0$ if $l<0$ and $\phi_{00 k}=0$ if $k<0$.

As discussed in $\S \mathrm{I}$, we are interested in the case in which $e_{s} \ll 1$ and $|\Delta a|=\left|a-a_{s}\right| \ll a_{s}$. Thus, the factor $\left(1-e_{s}^{2}\right)$ in front of the summation in equation (52) can be dropped. Similarly, we replace $m\left(1-e_{s}^{2}\right)^{-1 / 2}-$ $\omega / n_{s}=m\left(1-e_{s}^{2}\right)^{-1 / 2}-l$ by $\frac{1}{2} l e_{s}^{2}$ if $m=l$ and by $(m-l)$ if $m \neq l$. We also set $n_{s}=n$ and $a_{s}=a$ except in combinations involving the difference of $n_{s}$ and $n$. Finally, we define $q=l-k$. The resonance condition becomes $n-n_{s}=q n_{s} / k$. For $\left|n-n_{s}\right| \ll n_{s}, k \gg 1$, so the sum over $k$ is replaced by an integral. The integral is evaluated to be

$$
\begin{aligned}
& \frac{d e_{s}^{2}}{d t}=\frac{2 \pi}{n_{s}{ }^{2} a_{s}{ }^{4}}\left(\frac{m_{r}}{m_{s}}\right) \operatorname{sgn}\left(n-n_{s}\right) \llbracket \frac{1}{4} \frac{n_{s} e_{s}^{2}}{\left(n-n_{s}\right)^{2}}\left(\left.\frac{\partial \phi_{q+k, k-1, k}^{2}}{e \partial e}\right|_{q=-1}\right. \\
&\left.-\left.\frac{\partial \phi_{q+k, k+1, k}^{2}}{e \partial e}\right|_{q=1}\right)-\sum_{q=-\infty}^{\infty}\left\{n_{s} a_{s} q^{2} \frac{\partial}{\partial a}\left(\frac{\phi_{q+k, k, k}^{2}}{\left(n-n_{s}\right)^{2}}\right)\right. \\
&\left.\quad+\frac{1}{2} \frac{1}{\left(n-n_{s}\right)}\left[(1+q) \frac{\partial \phi_{q+k, k-1, k}^{2}}{e \partial e}+(1-q) \frac{\partial \phi_{q+k, k+1, k}}{e \partial e}\right]\right\} \|_{e=0} .
\end{aligned}
$$

In equation (53) $\phi_{l m k}$ is a function of $a$ and $e$, and $k$ is an implicit function of $a$ through the relation $k=q n_{s} /\left(n-n_{s}\right)$. Since $k \gg 1$, only terms with $\operatorname{sgn}(q)=\operatorname{sgn}\left(n-n_{s}\right)$ contribute.

\section{d) The Effect of Resonances on an Eccentric Ring}

We wish to describe the evolution of the eccentricity of an eccentric ring. Since the mean eccentricity vector $\langle\boldsymbol{e}\rangle$ is approximately conserved in collisions $(\S \mathrm{II} b)$, we need only calculate the change in $\langle\boldsymbol{e}\rangle$ due to gravitational perturbations from the satellite.

Denote first-and second-order changes by the subscripts 1 and 2 respectively. Denote $|\langle e\rangle|$ by $e_{r}$, the ring eccentricity. Then the second-order change in the ring eccentricity is

$$
\frac{d e_{r 2}}{d t}=\left\langle\frac{d e_{2}}{d t}\right\rangle-e\left\langle\varpi_{1} \frac{d \varpi_{1}}{d t}\right\rangle+\frac{1}{e}\left\langle e_{1}\right\rangle\left\langle\frac{d e_{1}}{d t}\right\rangle-e\left\langle\varpi_{1}\right\rangle\left\langle\frac{d \varpi_{1}}{d t}\right\rangle .
$$

For rings with $e \ll 1,\left\langle d e_{1} / d t\right\rangle=\left\langle d I_{1} / d t\right\rangle / n a^{2} e$, and $\left\langle d e_{2} / d t\right\rangle=\left\langle d I_{2} / d t\right\rangle / n a^{2} e-\left\langle I_{1} d I_{1} / d t\right\rangle /\left(n^{2} a^{4} e^{3}\right)$. Calculations analogous to those of $\S$ III $b$ yield

$$
\begin{aligned}
\left\langle\frac{d \varpi_{1}}{d t}\right\rangle & =0, \\
\left\langle\frac{d I_{1}}{d t}\right\rangle & =0, \\
\left\langle\frac{d I_{2}}{d t}\right\rangle_{\mathrm{L}} & =\frac{\pi}{2}(k-m)^{2} \frac{\partial \phi_{m k}^{2}}{\partial I} \delta(k n-\omega), \\
\left\langle\frac{d I_{2}}{d t}\right\rangle_{\mathrm{c}} & =\frac{\pi k(k-m)}{n a} \frac{\partial}{\partial a}\left[\phi_{m k}^{2} \delta(k n-\omega)\right], \\
\left\langle\varpi_{1} \frac{d \varpi_{1}}{d t}\right\rangle & =\frac{\pi}{2}\left(\frac{\partial \phi_{m k}}{\partial I}\right)^{2} \delta(k n-\omega), \\
\left\langle I_{1} \frac{d I_{1}}{d t}\right\rangle & =\frac{\pi}{2}(k-m)^{2} \phi_{k m}^{2} \delta(k n-\omega),
\end{aligned}
$$

where we have let $\gamma \rightarrow 0$. The subscripts $C$ and $\mathrm{L}$, as usual, denote corotation and Lindblad terms. The subscripts $C$ and $\mathrm{L}$ have not been used in equations (55e) and (55f); however, these terms are similar to Lindblad terms in that they are present unless there is a gap at the resonance, and they are included as parts of the Lindblad terms below.

Next we combine equations (54) and (55). We assume $e \ll 1$ and regard $\phi_{m k}$ as a function of $a$ and $e$. We find

$$
\begin{aligned}
\left\langle\frac{d e_{2 r}}{d t}\right\rangle_{\psi, \mathrm{L}} & =\frac{\pi}{2 n^{2} a^{4} e} \delta(k n-\omega)\left[\frac{(m-k)^{2}}{e} \frac{\partial \phi_{m k}^{2}}{\partial e}-(m-k)^{2} \frac{\phi_{m k}^{2}}{e^{2}}-\left(\frac{\partial \phi_{m k}}{\partial e}\right)^{2}\right], \\
\left\langle\frac{d e_{2 r}}{d t}\right\rangle_{\psi, c} & =\frac{\pi k(k-m)}{n^{2} a^{4} e} a \frac{\partial}{\partial a}\left[\phi_{m k}^{2} \delta(k n-\omega)\right] .
\end{aligned}
$$


We apply these results to a ring which is perturbed by a nearby satellite. It is assumed that the satellite moves on a circular orbit so that $\omega=m n_{s}$.

In the notation of the previous subsection $\phi_{m k}=\phi_{m m k}$, and for $m=0$ we have $\phi_{00 k}=0$ if $k<0$. Since $|\Delta a|=\mid a-$ $a_{s} \mid \ll a_{s}$, we set $n_{s}=n$ and $a_{s}=a$ except in combinations depending on the difference $n_{s}-n$. We define $q=m-k$, so the resonance condition becomes $n-n_{s}=q n_{s} / k$. Since $\left|n-n_{s}\right| \ll n_{s}$, the sum over $k$ is replaced by an integral. We evaluate the integral to obtain

$$
\begin{aligned}
& \left\langle\frac{d e_{2 r}{ }^{2}}{d t}\right\rangle_{\mathrm{L}}=\frac{\pi}{n_{s}{ }^{2} a_{s}{ }^{4}\left|n-n_{s}\right|} \sum_{q=-\infty}^{\infty}\left[\frac{q^{2}}{e} \frac{\partial \phi_{q+k, q+k, k}^{2}}{\partial e}-q^{2} \frac{\phi_{q+k, q+k, k}^{2}}{e^{2}}-\left(\frac{\partial \phi_{q+k, q+k, k}}{\partial e}\right)^{2}\right], \\
& \left\langle\frac{d e_{2 r}{ }^{2}}{d t}\right\rangle_{\mathrm{C}}=\frac{2 \pi \operatorname{sgn}\left(n_{s}-n\right)}{n_{s} a_{s}{ }^{3}} \sum_{q=-\infty}^{\infty} q^{2} \frac{\partial}{\partial a}\left[\frac{\phi_{q+k, q+k, k}^{2}}{\left(n_{s}-n\right)^{2}}\right] .
\end{aligned}
$$

In equation (57) the subscript $k$ is to be regarded as an implicit function of $a$ through $k=q n_{s} /\left(n-n_{s}\right)$. Since $k \gg 1$, only terms with $\operatorname{sgn}(q)=\operatorname{sgn}\left(n-n_{s}\right)$ contribute to the sum.

\section{SATELLITE POTENTIAL}

We evaluate the potential due to a satellite of mass $m_{s}$, semimajor axis $a_{s}$, and eccentricity $e_{s}$. We neglect precession of the satellite orbit, set $\varpi_{s}=0$, and choose $t_{s}=0$ for the time of periapse passage.

The gravitational potential from the satellite may be written as

$$
\phi(r, t)=G m_{s}\left[-\left|r-r_{s}(t)\right|^{-1}+\frac{r \cdot r_{s}(t)}{r_{s}^{3}(t)}\right],
$$

where $r_{s}=\left(r_{s}, \theta_{s}\right)$ is the position of the satellite at time $t$. Fourier expansion of $\phi$ in the angle $\theta-\theta_{s}$ yields

$$
\phi(r, \theta, t)=-\frac{G m_{s}}{r_{s}}\left[\frac{1}{2} \sum_{j=-\infty}^{\infty} b_{1 / 2}^{(j)}\left(\frac{r}{r_{s}}\right) \cos j\left(\theta-\theta_{s}\right)-\frac{r}{r_{s}} \cos \left(\theta-\theta_{s}\right)\right],
$$

where $b_{1 / 2}^{(j)}$ is a Laplace coefficient. Following equation (9), we write

$$
\phi(r, \theta, t)=\sum_{l=-\infty}^{\infty} \sum_{k=-\infty}^{\infty} \sum_{m=0}^{\infty} \phi_{l m k}(a, e) \cos \left[k \lambda+(m-k) \varpi-\ln _{s} t\right] .
$$

The coefficient $\phi_{l m k}$ is given by

$$
\phi_{l m k}=\frac{1}{4 \pi^{3}\left(1+\delta_{l 0} \delta_{m 0} \delta_{k 0}\right)} \int_{0}^{2 \pi} d \lambda \int_{0}^{2 \pi} d \varpi \int_{0}^{2 \pi} d\left(n_{s} t\right) \phi(r, \theta, t) \exp \left\{-i\left[k \lambda+(m-k) \varpi-\ln _{s} t\right]\right\} .
$$

In practice the term $l=m=k=0$ is never important, so we drop the factor $1+\delta_{l 0} \delta_{m 0} \delta_{k 0}$. Since $\varpi_{s}=0$ and $t_{s}=0, \phi(r$, $\theta, t)=\phi(r,-\theta,-t)$, and

$$
\phi_{l m k}=\frac{1}{4 \pi^{3}} \int_{0}^{2 \pi} d \lambda \int_{0}^{2 \pi} d \varpi \int_{0}^{2 \pi} d\left(n_{s} t\right) \phi(r, \theta, t) \cos \left[k \lambda+(m-k) \varpi-\ln _{s} t\right] .
$$

In terms of the mean anomaly $M=\lambda-\varpi$,

$$
\phi_{l m k}=\frac{1}{4 \pi^{3}} \int_{0}^{2 \pi} d M \int_{0}^{2 \pi} d \varpi \int_{0}^{2 \pi} d\left(n_{s} t\right) \phi(r, \theta, t) \cos \left(k M+m \varpi-\ln _{s} t\right) .
$$

Now write $\theta=\varpi+f(e, M)$; the function $f$ is defined in equation (65). Equations (59) and (63) yield

$$
\phi_{l m k}=-\frac{G m_{s}}{4 \pi^{2}} \int_{0}^{2 \pi} d M \int_{0}^{2 \pi} \frac{d\left(n_{s} t\right)}{r_{s}}\left[b_{1 / 2}^{(m)}\left(\frac{r}{r_{s}}\right)-\delta_{m, 1} \frac{r}{r_{s}}\right] \cos \left[k M-\ln _{s} t+m\left(\theta_{s}-f\right)\right] .
$$

We are interested in the case $m \gg 1$ and $e \ll 1$. Thus, we neglect the term involving $\delta_{m, 1}$ and write

$$
\begin{aligned}
r & =a(1-e \cos M), \\
f & =M+2 e \sin M, \\
r_{s} & =a_{s}\left(1-e_{s} \cos n_{s} t\right), \\
\theta_{s} & =n_{s} t+2 e_{s} \sin n_{s} t .
\end{aligned}
$$


We define $n_{s} t=M_{s}$ and substitute in equation (64) to arrive at

$$
\begin{aligned}
\phi_{l m k}=-\frac{G m_{s}}{4 \pi^{2} a_{s}} \int_{0}^{2 \pi} d M \int_{0}^{2 \pi} & d M_{s} b_{1 / 2}^{(m)}\left[\frac{a}{a_{s}}\left(1-e \cos M+e_{s} \cos M_{s}\right)\right] \\
& \times\left(1+e_{s} \cos M_{s}\right) \cos \left[(k-m) M-(l-m) M_{s}+2 m\left(e_{s} \sin M_{s}-e \sin M\right)\right] .
\end{aligned}
$$

Finally, since $\left|a-a_{s}\right| / a_{s} \ll 1$, it is convenient to use

$$
\begin{aligned}
b_{1 / 2}{ }^{(m)}(\alpha) & =\frac{2}{\pi} \int_{0}^{\pi} \frac{\cos m \phi d \phi}{\left(1-2 \alpha \cos \phi+\alpha^{2}\right)^{1 / 2}} \\
& \approx \frac{2}{\pi|\alpha-1|} \int_{0}^{\pi} \frac{\cos m \phi d \phi}{\left[1+\phi^{2} /(\alpha-1)^{2}\right]^{1 / 2}} \\
& \approx \frac{2}{\pi} K_{0}[|m(\alpha-1)|],
\end{aligned}
$$

where $K_{0}$ is a modified Bessel function.

We substitute equation (67) in equation (66), write $\Delta a=a-a_{s}$, and retain terms which contain $m e_{s}$ and $m e$ but drop those which contain $e$ and $e_{s}$ alone. The resulting expression is

$$
\begin{aligned}
\phi_{l m k}=-\frac{G m_{s}}{2 \pi^{3} a_{s}} \int_{0}^{2 \pi} d M \int_{0}^{2 \pi} d M_{s} K_{0}\left[\mid m\left(\Delta a / a_{s}\right.\right. & \left.\left.-e \cos M+e_{s} \cos M_{s}\right) \mid\right] \\
& \times \cos \left[(k-m) M-(l-m) M_{s}+2 m\left(e_{s} \sin M_{s}-e \sin M\right)\right] .
\end{aligned}
$$

The condition that the satellite and ring particle cannot collide for any orientation of the apse line is $|\Delta a|>a_{s}\left(e+e_{s}\right)$, which also guarantees the nonvanishing of the argument of the Bessel function.

\section{ECCENTRICITY EVOLUTION}

\section{a) Circular Ring and Eccentric Satellite Orbit}

The evolution of the satellite eccentricity is governed by equation (53). The required potential components come from equation (68). Since $|m-k| \leq 1$, we replace $m$ by $k$ wherever it is multiplied by $\Delta a / a_{s}$, $e$, or $e_{s}$ in equation (68), and we replace $k$ by $\Delta a$ using the resonance condition $k=q n_{s} /\left(n-n_{s}\right)=-2 q a_{s} / 3 \Delta a$. Finally, we define $\epsilon_{s} \equiv e_{s} a_{s} /|\Delta a|=$ $\frac{3}{2} e_{s} n_{s} /\left|n_{s}-n\right|$. Then

$$
\begin{array}{r}
\phi_{q+k, k, k}=-\frac{G m_{s}}{2 \pi^{3} a_{s}}(\operatorname{sgn} \Delta a)^{q} \int_{0}^{2 \pi} d M \int_{0}^{2 \pi} d M_{s} K_{0}\left[\frac{2|q|}{3}\left(1-\epsilon \cos M+\epsilon_{s} \cos M_{s}\right)\right] \\
\quad \times \cos \left[q M_{s}+\frac{4}{3} q\left(\epsilon_{s} \sin M_{s}-\epsilon \sin M\right)\right] . \\
\begin{aligned}
\phi_{q+k, k \pm 1, k}=-\frac{G m_{s}}{2 \pi^{3} a_{s}}(\operatorname{sgn} \Delta a)^{q} \int_{0}^{2 \pi} d M \int_{0}^{2 \pi} d M_{s} K_{0} & {\left[\frac{2|q|}{3}\left(1-\epsilon \cos M+\epsilon_{s} \cos M_{s}\right)\right] } \\
& \times \cos \left[q M_{s} \pm\left(M-M_{s}\right)+\frac{4}{3} q\left(\epsilon_{s} \sin M_{s}-\epsilon \sin M\right)\right] .
\end{aligned}
\end{array}
$$

For these expressions we can show that

$$
\left|\phi_{q+k, k, k}(e=0)\right|=\frac{2 G m_{s}}{\pi^{2} a_{s}}\left|F_{q}\left(\epsilon_{s}\right)\right|,
$$

where

$$
F_{q}\left(\epsilon_{s}\right)=\int_{0}^{\pi} d u K_{0}\left[\frac{2|q|}{3}\left(1+\epsilon_{s} \cos u\right)\right] \cos \left[|q|\left(u+\frac{4}{3} \epsilon_{s} \sin u\right)\right]
$$

Similarly, as $e \rightarrow 0$

$$
\left|\phi_{q+k, k \pm 1, k}\right| \rightarrow e \frac{G m_{s}}{\pi^{2} a_{s}} \frac{a_{s}}{|\Delta a|}\left|\frac{d F_{q}}{d \epsilon_{s}} \pm \frac{q}{\epsilon_{s}} F_{q}\right| .
$$

From these results we find that the term proportional to $e_{s}{ }^{2}$ in equation (53) is always small and can be dropped. Thus,

$$
\frac{d e_{s}^{2}}{d t}=\frac{1}{\pi^{3}} n_{s} \frac{m_{r} m_{s}}{M^{2}}\left|\frac{n_{s}}{n_{s}-n}\right|^{3} \sum_{q=1}^{\infty}\left\{-12 q^{2}\left(2 F_{q}^{2}+\epsilon_{s} d F_{q}^{2} / d \epsilon_{s}\right)+9\left[\left(q^{2} / \epsilon_{s}\right) d F_{q}^{2} / d \epsilon_{s}-q^{2} F_{q}^{2} / \epsilon_{s}^{2}-\left(d F_{q} / d \epsilon_{s}\right)^{2}\right]\right\}
$$


The first group of terms arises from corotation resonances and the second group from Lindblad resonances.

We defer the evaluation and discussion of this equation until the end of the next subsection.

\section{b) Eccentric Ring and Circular Satellite Orbit}

The evolution of the eccentricity of a ring is governed by equation (57). The required potential components come from equation (68). Since $|q| \ll k$, we have $\phi_{q+k, q+k, k} \approx \phi_{k, k, k-q}$. Also $k=q n_{s} /\left(n-n_{s}\right)=-2 q a_{s} / 3 \Delta a$. We define $\epsilon=e a_{s} /|\Delta a|=\frac{3}{2} e n_{s} /\left|n_{s}-n\right|$. We obtain

$$
\left|\phi_{q+k, q+k, k}\left(e_{s}=0\right)\right|=\frac{2 G m_{s}}{\pi^{2} a_{s}}\left|F_{q}(\epsilon)\right|,
$$

where $F_{q}$ is defined in equation (71). Equation (57) then becomes

$$
\begin{aligned}
& \left(\frac{d e^{2}}{d t}\right)_{\mathrm{L}}=\frac{1}{\pi^{3}} n_{s}\left(\frac{m_{s}}{M}\right)^{2}\left|\frac{n_{s}}{n_{s}-n}\right|^{3} \sum_{q=1}^{\infty} 9\left[\left(q^{2} / \epsilon\right) d F_{q}{ }^{2} / d \epsilon-q^{2} F_{q}{ }^{2} / \epsilon^{2}-\left(d F_{q} / d \epsilon\right)^{2}\right], \\
& \left(\frac{d e^{2}}{d t}\right)_{C}=\frac{1}{\pi^{3}} n_{s}\left(\frac{m_{s}}{M}\right)^{2}\left|\frac{n_{s}}{n_{s}-n}\right|^{3} \sum_{q=1}^{\infty} 12 q^{2}\left(-2 F_{q}{ }^{2}-\epsilon d F_{q}{ }^{2} / d \epsilon\right) .
\end{aligned}
$$

We drop the distinction between the symbols $e$ and $e_{r}$ for the ring eccentricity.

Apart from a factor $m_{s} / m_{r}$, equations (73) and (75) are identical. The eccentricity evolution of an eccentric satellite orbit under the influence of a circular ring is similar to the eccentricity evolution of an eccentric ring under the influence of a satellite which moves on a circular orbit. Thus, we confine future discussion to the evolution of an eccentric ring (eq. [75]).

We are particularly interested in the limit $\epsilon \rightarrow 0$ since it determines whether a circular ring is unstable to eccentricity growth. It is easy to see from equation (71) that as $\epsilon \rightarrow 0, F_{q}(\epsilon) \sim \epsilon^{|q|}$. Thus, resonances with small $|q|$ are most important. As $\epsilon \rightarrow 0$, the first two functions $F_{q}$ have the limiting forms

$$
\begin{aligned}
& F_{1}(\epsilon)=\frac{1}{3} \pi \epsilon\left[2 K_{0}\left(\frac{2}{3}\right)+K_{1}\left(\frac{2}{3}\right)\right]+O\left(\epsilon^{3}\right), \\
& F_{2}(\epsilon)=\frac{1}{18} \pi \epsilon^{2}\left[20 K_{0}\left(\frac{4}{3}\right)+19 K_{1}\left(\frac{4}{3}\right)\right]+O\left(\epsilon^{4}\right) .
\end{aligned}
$$

Thus, as $\epsilon \rightarrow 0$ equation (75) yields

$$
\begin{aligned}
& \left(\frac{d e^{2}}{d t}\right)_{\mathrm{L}}=+\frac{1}{2 \pi} e^{2}\left(\frac{m_{s}}{M}\right)^{2} \frac{n_{s}{ }^{6}}{\left|n_{s}-n\right|^{5}}\left[20 K_{0}\left(\frac{4}{3}\right)+19 K_{1}\left(\frac{4}{3}\right)\right]^{2}, \\
& \left(\frac{d e^{2}}{d t}\right)_{C}=-\frac{12 e^{2}}{\pi}\left(\frac{m_{s}}{M}\right)^{2} \frac{n_{s}{ }^{6}}{\left|n_{s}-n\right|^{5}}\left[2 K_{0}\left(\frac{2}{3}\right)+K_{1}\left(\frac{2}{3}\right)\right]^{2} .
\end{aligned}
$$

The Lindblad term tends to increase the ring eccentricity, while the corotation term tends to damp it. In the absence of other effects the corotation term in equation (77) is larger by a small margin $(\sim 5 \%)$, and the eccentricity damps. The numerical result in this case is (GT6)

$$
\frac{d e^{2}}{d t}=\left(\frac{d e^{2}}{d t}\right)_{\mathrm{L}}+\left(\frac{d e^{2}}{d t}\right)_{C}=-1.122\left(\frac{m_{s}}{M}\right)^{2} \frac{n_{s}{ }^{6}}{\left|n_{s}-n\right|^{5}} e^{2} .
$$

This result changes if the resonance torques saturate. In a nearly circular ring the strengths of all resonances with $|q|>1$ are much smaller than those with $|q|=1$. According to equations (38), (74), and (75), the Lindblad torque of a resonance with $|q|=1$ opens a gap if

$$
m_{s} / M \geq\left(v / n_{s} a_{s}^{2}\right)^{1 / 2}\left|n-n_{s}\right| / n_{s} .
$$

In the presence of a gap the $|q|=1$ resonance torques are largely shut off. The spacing between successive resonances of a given $q$ is proportional to $|q|^{-1}$. Between two $|q|=1$ resonances there is one $|q|=2$ resonance, two $|q|=3$ resonances, and so on. These particular resonances with $|q| \geq 1$ are unaffected by the gaps at the $|q|=1$ resonances.

Thus, if condition (79) is satisfied, the $|q|=1$ (corotation) terms in equation (77) which damp the eccentricity disappear, leaving half of the $|q|=2$ (Lindblad) terms (since half of the $|q|=2$ resonances are unaffected). The ring eccentricity then grows at a rate

$$
\frac{d e^{2}}{d t}=+11.563\left(\frac{m_{s}}{M}\right)^{2} \frac{n_{s}{ }^{6}}{\left|n_{s}-n\right|^{5}} e^{2} .
$$

We have established that if gaps form at the $|q|=1$ resonances, the eccentricity of a nearly circular ring grows. As the eccentricity grows, its evolution becomes more complicated because of the increasing importance of resonances with $|q| \gg 1$. The summands of equation (75) are plotted in Figure 1 as a function of $\epsilon$ for $q=1, \ldots, 5$. Although the influence of the $q=3$ resonance is negligible for $\epsilon \ll 1$, it contributes half of the total $d e / d t$ at $\epsilon \sim 0.1$. Its surprisingly strong 
contribution at $\epsilon=0.1$ is due to the near cancellation between the $|q|=1$ corotation torque and the $|q|=2$ Lindblad torque. Similarly, the $q=4$ resonances are important for $\epsilon \gtrsim 0.3$, again because of near cancellation.

A further complication is that, as Figure 1 shows, the resonance torques rapidly strengthen as $\epsilon \rightarrow 1$, and many resonances may saturate. If condition (39) is satisfied for a given resonance, the corotation terms do not contribute to the evolution, and if condition (38) is satisfied, neither the corotation nor Lindblad terms contribute.

Because of these complications, it is difficult to decide the ultimate fate of an eccentric ring once $\epsilon \sim O(1)$. The eccentricity growth may stop at some finite $\epsilon<1$, or growth may continue until the ring and satellite collide.

A further difficulty with our model is that the satellite orbit may be eccentric as well. It is straightforward to extend our formalism to the interactions of an eccentric ring and an eccentric satellite, but we shall not do so in this paper.

\section{APPLICATION TO THE $\epsilon$ RING}

The following parameters for the $\epsilon$ ring are well established observationally (Nicholson et al. 1978): $e=0.0078$, $a=51,284 \mathrm{~km}, \delta a=60 \mathrm{~km}, \delta e=7.2 \times 10^{-4}$. Here $\delta a$ and $\delta e$ are the widths of the ring in semimajor axis and eccentricity. Since the optical depth is of order unity, we take $n t_{\text {coll }} \sim 1$ (e.g., GT1). The velocity dispersion in the ring is

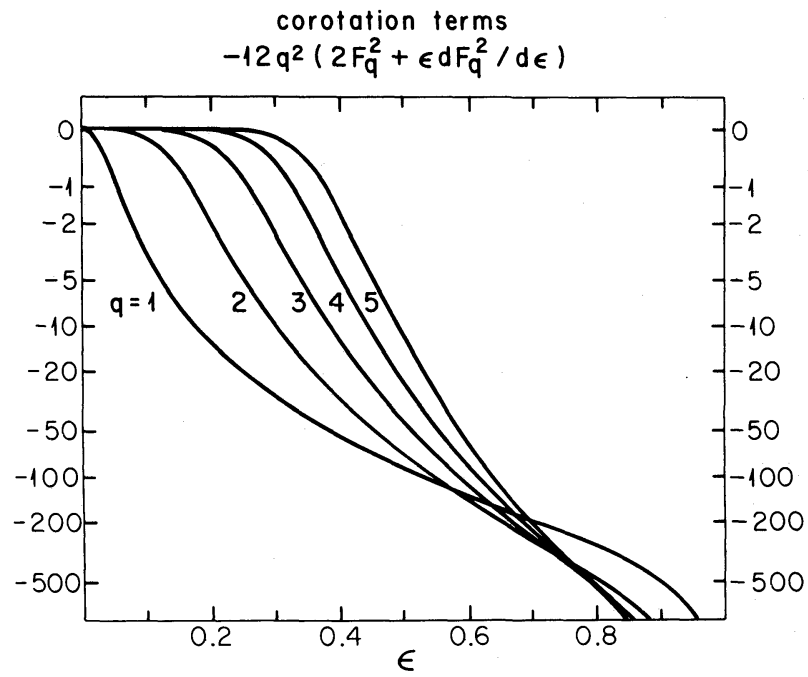

FIG. $1 a$

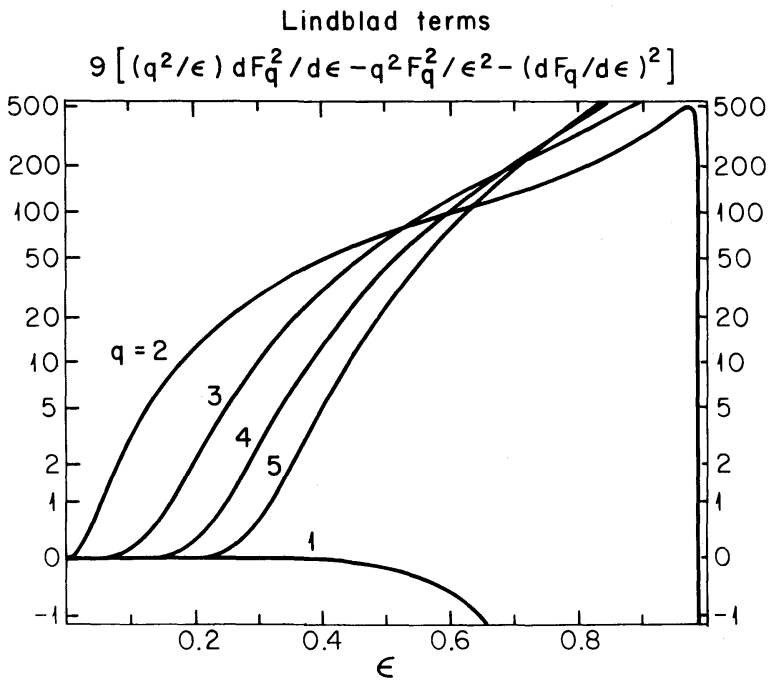

FIG. $1 b$

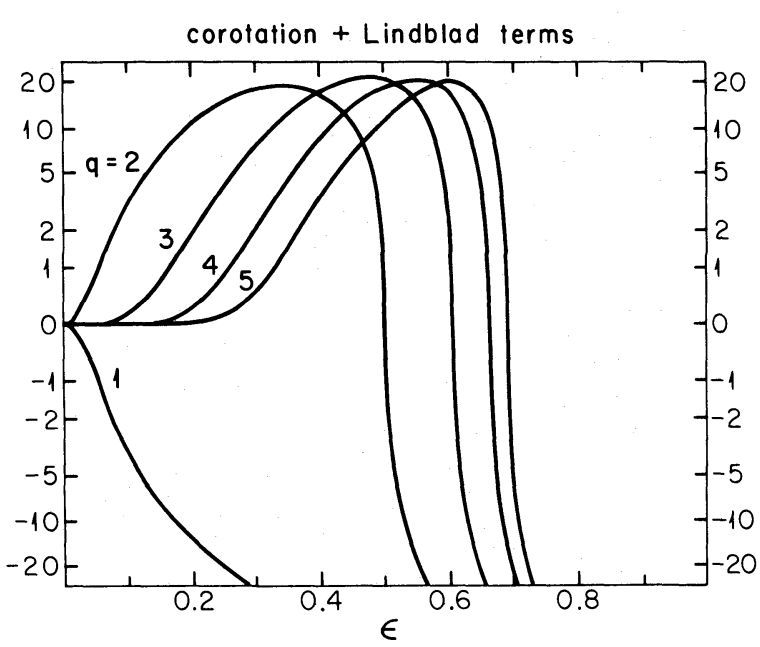

FIG. $1 c$

Fig. 1.- The summands of eqs. (73) and (75). Fig. $1 a$ shows the corotation terms $-12 q^{2}\left(2 F_{q}{ }^{2}+\epsilon d F_{q}{ }^{2} / d \epsilon\right)$, Fig. $1 b$ shows the Lindblad terms $9\left[\left(q^{2} / \epsilon\right) d F_{q}{ }^{2} / d \epsilon-q^{2} F_{q}{ }^{2} / \epsilon^{2}-\left(d F_{q} / d \epsilon\right)^{2}\right]$, and Fig. $1 c$ shows the sum of both terms. Note the near cancellation between the corotation terms of order $q$ and the Lindblad terms of order $q+1$ for small $\epsilon$. To accommodate a larger range the $y$-axis has been stretched, using the mapping $y \rightarrow \sinh y$. 
much less certain. We shall take $c \sim 10^{-2} \mathrm{~cm} \mathrm{~s}^{-1}$, roughly appropriate for a monolayer of surface density $\sim 20 \mathrm{~g} \mathrm{~cm}^{-2}$ (GT3). The resulting viscosity $v$ is roughly $0.2 c^{2} / n, \sim 0.1 \mathrm{~cm}^{2} \mathrm{~s}^{-1}(c / 0.01)^{2}$. We proposed that satellites of mass $m_{s} \sim 10^{19} \mathrm{~g}$ exist at distances $\Delta a \sim 500 \mathrm{~km}$ from the $\epsilon$ ring. Such satellites could constrain the ring against spreading from interparticle collisions and the Poynting-Robertson effect. The value of $k$ for a resonance with $|q|=1$ lying in the ring is $|k|=68|500 \mathrm{~km} / \Delta a|$. The distance between successive $|q|=1$ resonances is $7 \mathrm{~km}(\Delta a / 500 \mathrm{~km})^{2}$. Thus, there are several resonances within the ring, and our approximation of $k$ as a continuous variable is only partially justified.

A gap will open at a resonance with $|q|=1$ if (cf. eq. [79])

$$
m_{s} \gtrsim 1 \times 10^{19} \mathrm{~g}\left(\frac{c}{0.01 \mathrm{~cm} \mathrm{~s}^{-1}}\right)\left|\frac{\Delta a}{500 \mathrm{~km}}\right| .
$$

Our confinement theory predicts that the satellites associated with the $\epsilon$ ring have masses $\sim 10^{19} \mathrm{~g}$, which is very close to the minimum satellite mass required to open gaps. A possible reason for this near coincidence is that the masses of the confining satellites exceed $10^{19} \mathrm{~g}$, but because they open gaps at the resonances, the torques they exert on the ring are comparable in strength to the unsaturated torques from satellites with $m_{s} \sim 10^{19} \mathrm{~g}$ and $|\Delta a| \sim 500 \mathrm{~km}$.

Whether or not this speculation is correct, equation (81) shows that a satellite with reasonable mass $m_{s}$ and separation $\Delta a$ can open gaps. If these gaps are present, a circular ring is unstable, and we have a natural explanation for the eccentricity of the $\epsilon$ ring.

It is possible that some of the radial structure seen in the $\epsilon$ ring is the result of a small number of discrete resonances in the ring.

Some of the narrower Uranian rings appear to be circular. A possible explanation is that they are constrained by less massive satellites $\left(m_{s} \sim 10^{18} \mathrm{~g}\right.$, cf. GT3) which do not open gaps.

\section{SUMMARY AND GUIDE}

There are two kinds of important satellite resonances in a circular ring: corotation resonances and Lindblad resonances.

Perturbations in semimajor axis and mean longitude are excited near corotation resonances. Orbital eccentricities are not. Interparticle collisions damp the perturbations in semimajor axis and mean longitude, and there is a torque on the ring given by equation (32). If the resonant potential perturbation is sufficiently strong (cf. eq. [25]), the damping is incomplete, and the torque is reduced.

At Lindblad resonances the perturbing potential excites the eccentricities of the ring particles. Collisions between ring particles damp the eccentricities. As a result of this damping process, a torque is exerted on the ring in the vicinity of the resonance. If the torque is sufficiently weak, its magnitude is independent of the details of the damping process and is given by equation (36). If the resonance is sufficiently strong, a gap is opened at the resonance, and the torque is reduced. The condition for a gap to open is expressed in equation (38).

If the ring is eccentric, there is only one kind of resonance, which we have called an "eccentric" resonance. Collisional damping of perturbations near this resonance gives rise to a torque on the ring. The "corotation" terms arise from perturbations in $a$ and $\lambda$ and yield a torque similar to the one at a corotation resonance in a circular ring (cf. eq. [47]). The "Lindblad" terms arise from perturbations in $I$ and $\varpi$ and lead to a torque similar to the one which acts at a Lindblad resonance in a circular ring (eq. [44]). If the resonance is sufficiently strong, the corotation terms may saturate so that only the Lindblad terms are present, or else a gap may open so that both kinds of torque are reduced.

The evolution of the orbital eccentricity of a satellite near a circular ring is identical to the evolution of the eccentricity of a ring near a satellite which moves on a circular orbit (cf. eqs. [73] and [75]). For small eccentricities, the $q=1$ corotation terms damp the eccentricity, while the $q=2$ Lindblad terms excite it. In the absence of saturation, the corotation terms win by a small margin, and the eccentricity damps. If gaps open at the $q=1$ resonances, then the $q=2$ Lindblad terms win, and the eccentricity grows.

This research was supported in part by the National Science Foundation grants AST 79-24978 and PHY 79-19884 and NASA grant NGL-05-002-003.

Donner, K. 1979, Ph.D. thesis, University of Cambridge. Goldreich, P., and Tremaine, S. 1978a, Icarus, 34, 227 (GT1).

- 1978b, Icarus, 34, 240 (GT2). 1979a, Nature, 277, 97 (GT3) 1979b, Ap. J., 233, 857 (GT4).

\section{REFERENCES}

Goldreich, P., and Tremaine, S. 1979c, A.J., 84, 1638 (GT5). 1980, Ap. J., in press (GT6).

Lynden-Bell, D., and Kalnajs, A. J. 1972, M.N.R.A.S., 157, 1.

Nicholson, P. D., Persson, S. E., Matthews, K., Goldreich, P., and Neugebauer, G. 1978, A.J., 83, 1240.

Peter Goldreich: Mail Code 170-25, California Institute of Technology, Pasadena, CA 91125

Scott Tremaine: School of Natural Sciences, Institute for Advanced Study, Princeton, NJ 08540 